\title{
Hydroxyapatite extracted by animal bone image analysis in ionic liquid choline chloride-glycerol
}

\author{
Hong Zhu', Wei Song ${ }^{2 *}$ and Yu Deng ${ }^{3}$
}

\begin{abstract}
In this paper, the dissolving process of animal bone (chicken bone) in ionic liquid (IL) choline chloride-glycerol (ChCl-GL) was researched, and the product hydroxyapatite (HAp) was extracted. The optimum conditions of extracted HAp were determined by single factor, orthogonal, and verification tests. Based on dissolving situation of observing bone power in the IL by means of thermal stage polarized optical microscope (HSPOM), it could be preliminarily concluded that this dissolving process was a direct physical dissolution. IR analyses, which was for undissolved bone, under different solution temperature and time, showed that bone collagen was dissolved mainly in the IL. This was consistent with HSPOM observations. The change of internal hydrogen bond was an important factor of $\mathrm{IL}$ dissolving bone. IR characterization indicated that the macroscopic composition and microstructure of the extracted HAp had characteristics of natural bone. XRD and SEM displayed that the product HAp was much similar to a standard sample, and its crystal structure was relatively complete; moreover, its purity is higher than the sample's. The BET tests exposed that specific surface area and aperture of the extracted HAp were similar to the values of the sample, which belonged to the mesoporous. Analysis of $\mathrm{N}_{2}$ adsorption-desorption revealed that the extracted HAp had a significant specific surface area, so as to be used for different adsorption processes. TG characterization exposited that pyrolysis temperature of the product HAp was higher, so it had better stability.
\end{abstract}

Keywords: Dissolving, Extracted, Collagen, Characterization, BET (Brunauer-Emmett-Teller)

\section{Introduction}

With the rapid development of biological materials science, the researchers find that inorganic biomaterials, which are close to the composition of natural bone, have good biocompatibility and human-body adaptability. It is the ideal bone substitute and can be used as a filling medium material of bone damage. So, there is a very good application prospect in the medical field [1, 2]. Among them, hydroxyapatite (HAp), as a new biological material, is the most widely used in the fields of researches and application [3, 4]. HAp belongs to a surface active material and is the main inorganic component in natural bone. It is similar to natural bone not only on the macrostructure, but on microstructure, chemical composition, features,

\footnotetext{
* Correspondence: sw8322@126.com

${ }^{2}$ Tianjin Key Laboratory for Advanced Mechatronic System Design and Intelligent Control, School of Mechanical Engineering, Tianjin University of Technology, No. 391 Binshuixi Road, Tianjin 300384, China

Full list of author information is available at the end of the article
}

the good biocompatibility, organization activity, and bone induced. HAp does not contain other toxic elements and can provide necessary elements of calcium and phosphorus to the body's tissues. After being implanted in human body, it has no immunity or rejection effect on the body tissue. So, it can be used as a bone defect repair material to give full play to the role of bone conduction [5, 6]. The breakthrough research of tissue engineering and biomaterials cannot be done without nano-HAp. At present, the nanometer HAp has been very important in various fields. It is a target of the present day how to choose the preparation technique of different patterns HAp, and apply it to biomedical field [7, 8]. As the emergence of new biological materials hydroxyapatite, the research and utilization of cattle bone (including chicken bone, pig bone, cow bone, etc.) will be gradually turned to the extraction and application of hydroxyapatite in natural bone from extracting bone oil, bone glue and hydrolyzed gelatin, etc. Recently, people have already begun to seek 
the preparation and extraction process of natural HAp in order to respond to the "green chemistry," which the world is advocating. It has become a focused issue for the researchers to obtain HAp by choosing a green and efficient solvent to dissolve animal bones and other materials $[9,10]$. The ionic liquid (IL), as a veritable environment-friendly green solvent, shows the advantages of "green and pollution-free" compared with traditional organic solvents and is a kind of designability new green organic solvent and catalyst. ILs, as green solvents, have an excellent solubility property, which can dissolve most inorganic materials, organics, polymers, etc. [11-13]. ILs are non-volatile liquid solvents with a low melting point and have many practical applications, such as widely used in organic synthesis, catalysis, separation and purification, electrochemistry, and nanometer materials. So, there is a broad application prospect and it is expected to be an effective alternative to traditional organic solvent. In addition, since ILs possess a strong ability to break hydrogen bonds, they have been used to dissolve natural polymer materials which contain a large number of hydrogen bonds and macromolecular materials in terms of natural extraction, especially the extraction of protein $[14,15]$.

As reported, related work by Phillips et al. [16] made the silk bundle protein dissolved in a variety of ionic liquids and contrasted their dissolving status. They found that protein fiber could be better dissolved in the ionic liquid containing chlorine ions because this IL had the stronger ability of breaking hydrogen bond of biological macromolecules. Ren et al. [17] used 1-butyl3-methylimidazole acetate ionic liquid to dissolve the silk fibroin protein, and found that the maximum dissolved amount of silk fibroin in ionic liquid could reach 15\%. Zheng [18] found that the choline chloride IL has a certain solubility property for protein, and pointed out that collagen dissolving rate in choline chloride- $2 \mathrm{ZnCl} 2$ ionic liquid was affected significantly by temperature and increased with temperature. Yuan et al. [19] used choline chloride-urea ionic liquid to dissolve the chicken bones. They found that chicken bone solubility in the ionic liquid could reach $38 \%$, and predicted this process was a physical dissolve and the dissolved product was chicken bone hydroxyapatite.

Along with HAp, it is more and more widely used in the application of bionic composite materials, and the extraction of natural HAp will also be further development. On the one hand, it can take advantage of a large

Table 1 The effects of different solid-to-liquid ratios

\begin{tabular}{llllll}
\hline S/L ratios/g/g & $1: 10$ & $1: 20$ & $1: 30$ & $1: 40$ & $1: 50$ \\
Dissolving rate/\% & 23.12 & 28.41 & 32.64 & 31.52 & 26.54 \\
HAp yields/\% & 9.25 & 9.69 & 10.84 & 10.71 & 10.52 \\
\hline
\end{tabular}

Table 2 The effects of different dissolving time

\begin{tabular}{llllll}
\hline Time/h & 6 & 7 & 8 & 9 & 10 \\
Dissolving rate/\% & 32.64 & 34.11 & 35.13 & 35.15 & 35.16 \\
HAp yields/\% & 10.84 & 12.75 & 13.69 & 13.61 & 13.64 \\
\hline
\end{tabular}

number of abandoned animal bones; on the other hand, natural extracts of hydroxyapatite are closer to the human body skeleton compared with the chemical synthesis in physical properties, chemical properties, and biocompatibility. So, there will be a broader clinical application in the field of medical orthopedic and expected it to become a new type of bone injury repair material. In addition, the theoretical study of ionic liquid dissolving animal bones can enrich the understanding of ionic liquid and enlarge the application range of ionic liquid. The specific performance is as follows: (1) the ionic liquids can dissolve bone collagen, so as to achieve the aim of separation and hydroxyapatite collagen [20]. That will improve the comprehensive utilization of animal bone, and at the same time to expand the application range of new biological materials hydroxyapatite. (2) The purity of extracted HAp is higher than synthetic HAp, which possess macroscopic composition, microscopic structure of natural bone, and good biocompatibility. HAp composite material with organic components can be used in medical field of bone defects repair. Ionic liquids as a new green solvent can be recycled, which is conducive to protect the environment, in line with the concept of green chemistry. This method has a great prospect of application and the space for development in extracting HAp from livestock bone. This research intends to find a preparation method of natural HAp, which is closer to the natural human bone HAp than the chemical synthesis HAp on the physical properties, chemical properties, and biocompatibility. And this conditions are extended to extract product HAp by dissolving chicken bone in ChCl-GL (IL). In this paper, the mechanism of bone dissolution was analyzed, and product HAp was extracted and characterized by FTIR, SEM, XRD, BrunauerEmmett-Teller (BET), and TG.

\section{Method}

\subsection{Materials}

Choline chloride $\left(\mathrm{ChCl}, \mathrm{C}_{5} \mathrm{H}_{14} \mathrm{ClNO}\right)$ of food grade was provided by China Tianjin Shiyuan biotechnology Co. Ltd; glycerol (GL, $\mathrm{C}_{3} \mathrm{H}_{8} \mathrm{O}_{3}$ ) and methanol of

Table 3 The effects of dissolving temperature

\begin{tabular}{llllll}
\hline Temperature $/{ }^{\circ} \mathrm{C}$ & 80 & 100 & 120 & 140 & 160 \\
Dissolving rate/\% & 24.06 & 27.19 & 35.13 & 36.12 & 36.87 \\
HAp yields/\% & 12.97 & 13.16 & 13.69 & 13.53 & 14.32 \\
\hline
\end{tabular}


Table 4 The orthogonal factor level

\begin{tabular}{llll}
\hline & Time/h & Temperature $/{ }^{\circ} \mathrm{C}$ & S-L ratio/g/g \\
\hline 1 & 8 & 120 & $1: 30$ \\
2 & 8.5 & 130 & $1: 35$ \\
3 & 9 & 140 & $1: 40$ \\
\hline
\end{tabular}

analytical regent (AR) was provided by China Tianjin chemical reagent factory; hydroxyapatite (HAp, $\left.\mathrm{Ca} 10\left(\mathrm{PO}_{4}\right)_{6}(\mathrm{OH}) 2\right)$ of medical grade, medical hydroxyapatite powder (a synthesized material), was provided by China Xi'an Ruiying biotechnology Co. Ltd, and chosen as a standard sample.

Lab preparation: IL (ChCl-GL): $\mathrm{ChCl}$ and $\mathrm{GL}$ of 1:2 (mole) were added to a 3-mouth flask, which was placed in a thermostatic waterbath of $80{ }^{\circ} \mathrm{C}$. It was stirred until the solid disappears and form a deep eutectic transparent liquid. Then, keep heating for $1 \mathrm{~h}$, ChCl-GL(IL), which was clear and transparent [21]. It was transferred to a conical bottle. The equation of synthesis $\mathrm{ChCl}-\mathrm{GL}(\mathrm{IL})$ is as follows:

$$
\begin{aligned}
& {\left[\mathrm{HOC}_{2} \mathrm{H}_{4} \mathrm{~N}\left(\mathrm{CH}_{3}\right)_{3}\right]^{+}[\mathrm{Cl}]^{-}} \\
& +2 \mathrm{CHOH}\left(\mathrm{CH}_{2} \mathrm{OH}\right)_{2} \Leftrightarrow\left[\mathrm{HOC}_{2} \mathrm{H}_{4} \mathrm{~N}\left(\mathrm{CH}_{3}\right)_{3}\right]^{+} \\
& +\left[\mathrm{CHOH}\left(\mathrm{CH}_{2} \mathrm{OH}\right)_{2}\right]_{2}[\mathrm{Cl}]^{-}
\end{aligned}
$$

Chicken bone powder (40-60 mesh): the meat residue, fat, and connective tissue on fresh bones were removed after cleaning with clear water. The bones were soaked with gasoline for a period of time, then the grease was taken out by extraction solvent. The treated bones were washed again and dried. Finally, they were cut up to small pieces about $3 \mathrm{~cm}$ long and $1 \mathrm{~cm}$ in width, which the bone marrow was removed. Bone powder was obtained by smashing about 5 min with a high-speed multi-purpose pulverizer.

\subsection{HAp extracted by the IL dissolving chicken bone}

The bone powder and IL were put into a reaction container with a hot magnetic stirring. After mixing evenly, the experimental temperature was adjusted. Undissolved bone was obtained by filtering the mixture with a copper network (100 mesh aperture) after finishing the dissolving experiment. Then, the undissolved bone was transferred to a $500 \mathrm{ml}$ beaker and washed a few times with clear water. The IL adhering to undissolved bone would be washed off. After that, it was filtered by vacuum pump and dried in a drying oven at $70{ }^{\circ} \mathrm{C}$ for $24 \mathrm{~h}$. It was an undissolved bone sample.

The filtrate filtered by copper mesh was a mixture of IL, chicken bone collagen, and HAp. HAp of the mixture was precipitated priority by adding dilute methanol of 1:1 volume ratio, which also acted as a precipitant. Then, the grading filtering (normal filter paper + $450 \mathrm{~nm}+220 \mathrm{~nm}$ filter membrane) was conducted. The filter cake was washed repeatedly with distilled water to remove surface adhesion IL and dried at same condition. The precipitated accordingly was designated as the ex-

\begin{tabular}{|c|c|c|c|c|c|c|}
\hline & & S-L ratio/g/g & Temperature $/{ }^{\circ} \mathrm{C}$ & Time/h & Dissolving rate/\% & HAp yields $/ \%$ \\
\hline 1 & & $1: 30$ & 120 & 8 & 32.12 & 17.52 \\
\hline 2 & & $1: 30$ & 130 & 8.5 & 30.15 & 16.09 \\
\hline 3 & & $1: 30$ & 140 & 9 & 36.17 & 21.74 \\
\hline 4 & & $1: 35$ & 120 & 8.5 & 35.13 & 20.95 \\
\hline 5 & & $1: 35$ & 130 & 9 & 31.11 & 17.21 \\
\hline 6 & & $1: 35$ & 140 & 8 & 27.07 & 15.24 \\
\hline 7 & & $1: 40$ & 120 & 9 & 34.15 & 18.51 \\
\hline 8 & & $1: 40$ & 130 & 8 & 33.09 & 17.66 \\
\hline 9 & & $1: 40$ & 140 & 8.5 & 28.08 & 15.84 \\
\hline \multirow[t]{4}{*}{ Dissolving rate/\% } & K1 & 98.44 & 101.4 & 92.28 & - & - \\
\hline & $\mathrm{K} 2$ & 93.31 & 94.35 & 93.36 & - & - \\
\hline & K3 & 95.32 & 91.32 & 101.43 & - & - \\
\hline & $\mathrm{R}$ & 5.13 & 10.08 & 9.15 & - & - \\
\hline \multirow[t]{4}{*}{ HAp yields/\% } & $\mathrm{K} 1$ & 55.35 & 56.98 & 50.42 & - & - \\
\hline & $\mathrm{K} 2$ & 53.4 & 50.96 & 52.88 & - & - \\
\hline & K3 & 52.01 & 52.82 & 57.46 & - & - \\
\hline & $\mathrm{R}$ & 3.34 & 6.02 & 7.04 & - & - \\
\hline Optimal conditions & & $1: 30$ & 120 & 9 & - & - \\
\hline
\end{tabular}
tracted product HAp.

Table 5 The orthogonal experiments 
Table 6 The repeatability tests and results

\begin{tabular}{llllll}
\hline & Time/h & Temperature $/{ }^{\circ} \mathrm{C}$ & $\begin{array}{l}\mathrm{S} / \mathrm{L} \text { ratio } \\
\text { /g/g }\end{array}$ & $\begin{array}{l}\text { Dissolving } \\
\text { rate/\% }\end{array}$ & $\begin{array}{l}\text { HAp yields/ } \\
\%\end{array}$ \\
\hline 1 & 9 & 120 & $1: 30$ & 38.16 & 22.31 \\
2 & 9 & 120 & $1: 30$ & 38.15 & 21.96 \\
3 & 9 & 120 & $1: 30$ & 38.14 & 22.19 \\
\hline
\end{tabular}

The dissolving situation of bone in the IL was observed by thermal stage polarized optical microscope (HSPOM), which photos were taken every $15 \mathrm{~min}$ to make a comparison. The changes of undissolved bone were analyzed by FTIR under different solution temperature and time.

\subsection{Performance characterization of extracted HAp}

1. FTIR: infrared spectra of sample HAp and extracted HAp were obtained by using $\mathrm{KBr}$ pellets and recorded on NICOLET6700 Fourier infrared spectrometer (imported from America), the instrument resolution of $4 \mathrm{~cm}^{-1}$, the scanning number is 16 , the scanning range is $400 \sim 4000 \mathrm{~cm}^{-1}$.

2. XRD: the phase purity and crystallinity of extracted HAp and sample HAp was analyzed by a TD-3500 $\mathrm{X}$-ray powder diffractometer from Dandong Tongda Technology Co., Ltd, China.

3. SEM: the crystalline forms of extracted HAp were observed by SEM (JSM-6380LV, imported from Japanese) scanning.

4. Bulk density: the powder of extracted HAp was put in a clean, dry graduated cylinder $(10 \mathrm{ml})$, which was weighed by an analytical balance. Then, vibrating it 20 times on the table made the powder impaction, and shaking it to the surface smooth. Corresponding volume could be read directly. Bulk density could be calculated based on the average value of three parallel measurements.

5. BET characterization: specific surface area of extracted HAp was determined by the BET method based on adsorption and desorption of N2. The product was outgassed in a vacuum at $120{ }^{\circ} \mathrm{C}$ before adsorption and desorption of $\mathrm{N} 2$, then its average pore diameter and pore volume were identified. Specific surface area was calculated by the BET equation.

\subsection{Recycle IL}

Chlorinated choline and glycerol are easy to absorb water, so the $\mathrm{ChCl-GL(IL)} \mathrm{belongs} \mathrm{to} \mathrm{the} \mathrm{hydrophilic}$ ionic liquid. The IL contained the collagen dissolved, a small amount of unprecipitated hydroxyapatite and some absorbed water when the experiment finished. The IL was recycled with a rotary evaporator and the recycle rate was calculated (as following formula) after removing the impurities contained, according to the characteristics of low steam pressure of ILs.

$$
W=\frac{m}{m_{0}} \times 100 \%
$$

Where $W$ is the recycle rate of IL (\%), $M$ is the amount of IL recovered (g), and MO is the amount of IL added (g).

\section{Discussion and experimental results}

3.1 The optimum conditions of HAp extracted

\subsubsection{Single-factor experiments}

Effects of solid-to-liquid (S/L) ratio: adjust $\mathrm{pH}$ to 9.5, temperature $120{ }^{\circ} \mathrm{C}$, and dissolving time $6 \mathrm{~h}$. In the experimental process, different $\mathrm{S} / \mathrm{L}$ ratio $(\mathrm{g} / \mathrm{g})$ of chicken bone power to IL were changed. The results were shown in Table 1.

Effects of dissolving time: under $\mathrm{S} / \mathrm{L}$ ratio $1: 30(\mathrm{~g} / \mathrm{g})$, $\mathrm{pH} 9.5$, temperature $120{ }^{\circ} \mathrm{C}$, the effects of dissolving time were shown in Table 2.

Effects of dissolving temperature: under $\mathrm{S} / \mathrm{L}$ ratio 1:30(g/g), $\mathrm{pH} \mathrm{9.5,} \mathrm{dissolving} \mathrm{time} 8 \mathrm{~h}$, the effects of dissolving temperature were shown in Table 3.

\subsubsection{Orthogonal experiments}

The orthogonal experimental factors were designed in Table 4 according to the results of single-factor experiments. Therefore, the orthogonal experiments was conducted on the basis of these factors and levels. The tests data were shown in Table 5.

\subsubsection{Validation experiments}

Parallel verification experiments were conducted three times under above optimum conditions. The results

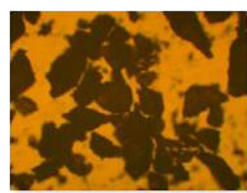

$15 \mathrm{~min}$

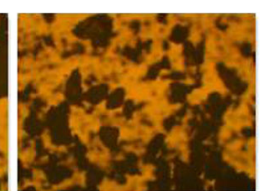

$30 \mathrm{~min}$

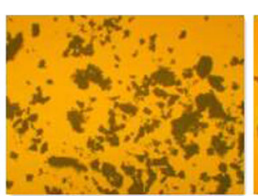

$45 \mathrm{~min}$

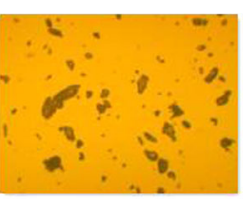

$60 \mathrm{~min}$

Fig. 1 HSPOM pictures of IL dissolving chicken bone 


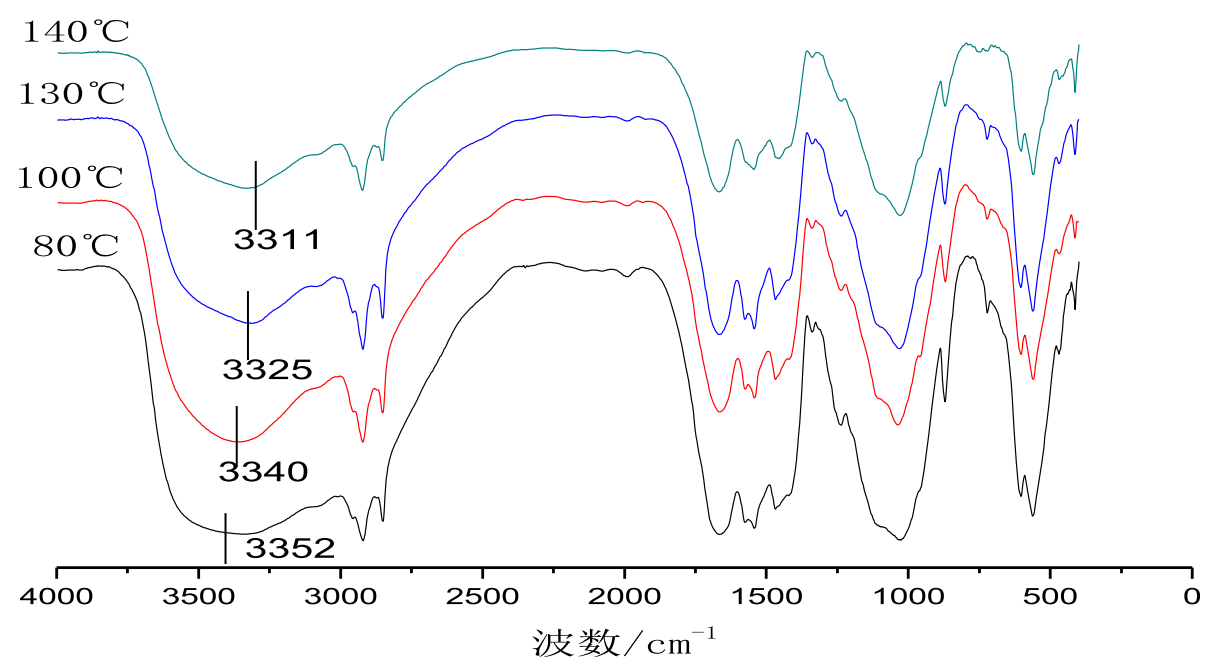

Fig. 2 IR spectra of undissolved bone at different temperature

were shown in Table 6. It endorsed that the reproducibility of optimum conditions were better.

\subsection{Discussion on the mechanism of IL (ChCl-GL) dissolving bone}

\subsubsection{HSPOM analysis}

The dissolving situation of bone powder in the IL was observed by means of HSPOM after experiment beginning under above optimal conditions (see Fig. 1). It could be seen that chicken bone was dissolved gradually in IL as time growth. It indicated that the IL had a certain solubility to some substances of bone. It would be preliminarily concluded that this dissolving process was a direct physical dissolution, and the dissolution product was the chicken bone HAp.

\subsubsection{FTIR analysis of undissolved bone at different temperatures}

Figure 2 showed infrared spectra of undissolved bone at different dissolution temperatures. It could been seen that $\mathrm{N}-\mathrm{H}$ stretching vibration peak at $3300 \mathrm{~cm}^{-1}$ moved to the direction of high wavelength with the increase of temperature, which the spectra occurred at the spectroscopic redshift. And the higher the temperature was, the more apparent the displacement was. The effect of IL on the hydrogen bonding interaction between peptide chains of bone collagen became obvious as the dissolution temperature rises, which had a marked impact on three-dimensional structure of collagen. Changing temperature made the change of characteristic peak of collagen amide zone more obvious. This revealed that the higher dissolution temperature enhanced the

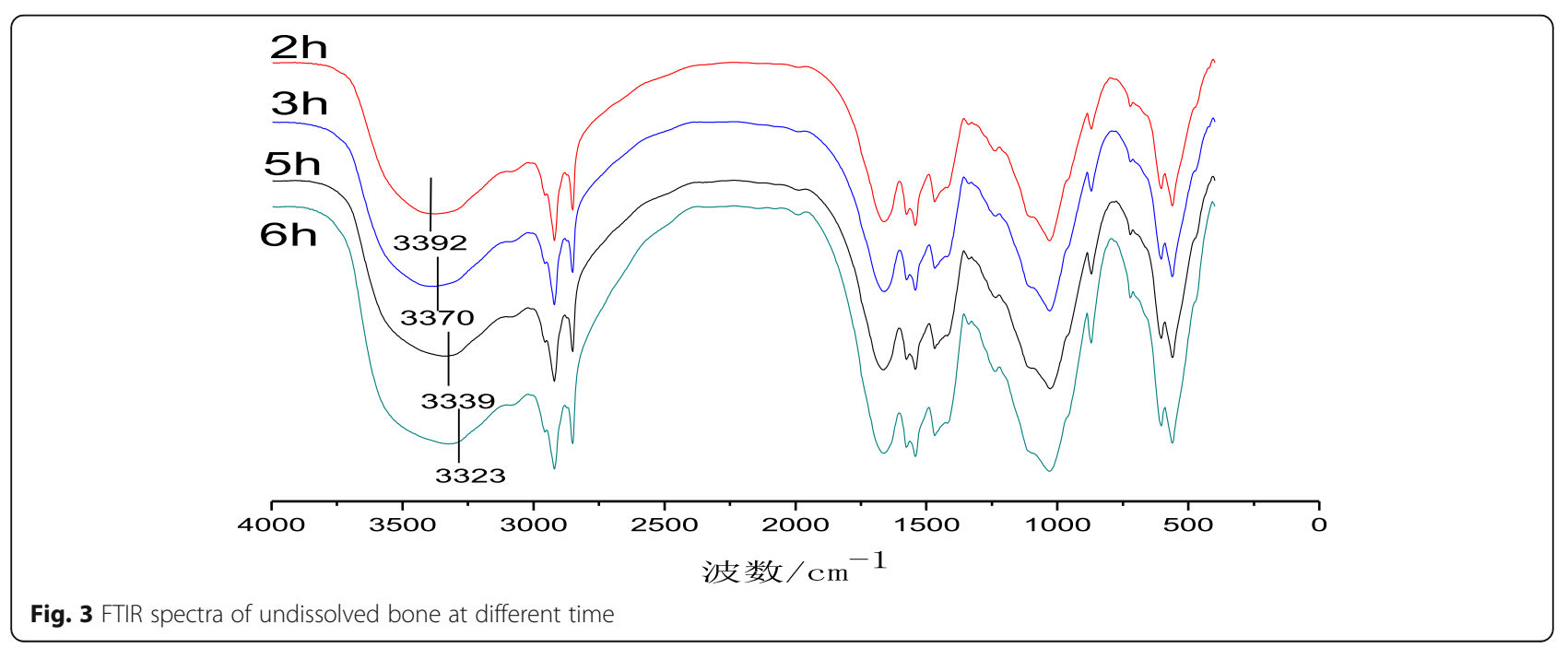




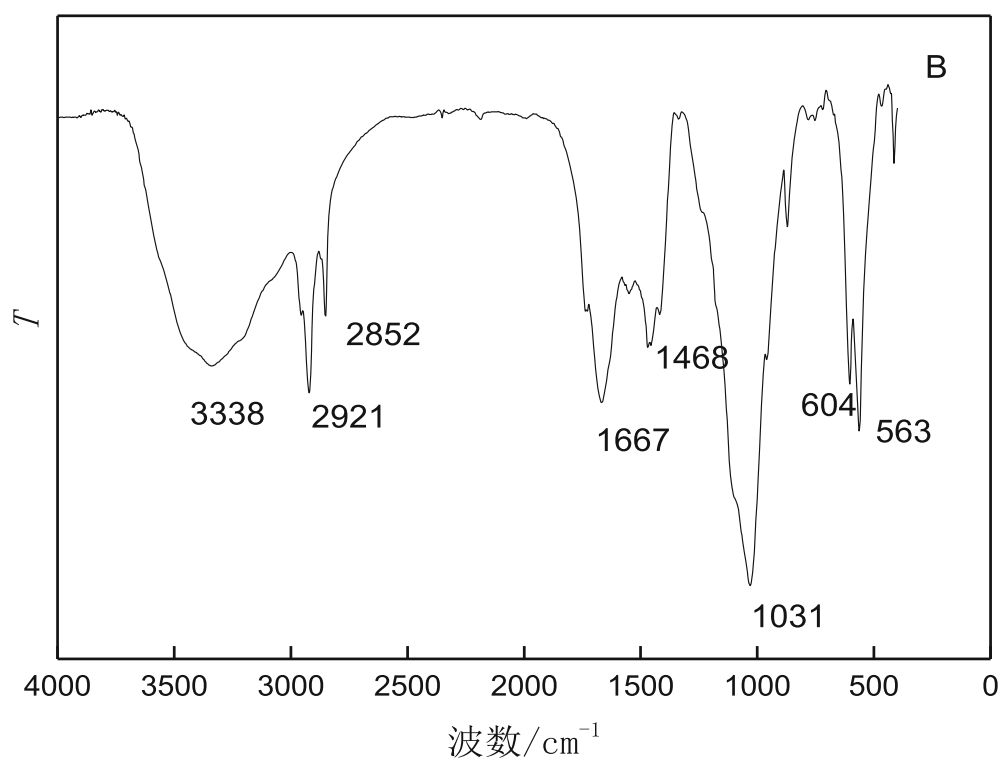

Fig. 4 IR spectrum of extracted HAp

influence of the IL on the hydrogen bond and molecular groups in peptide chain of bone collagen.

\subsubsection{IR analysis of undissolved bone at different dissolution time}

Figure 3 showed infrared spectra of undissolved bone at different dissolution time. It could been seen that $\mathrm{N}-\mathrm{H}$ stretching vibration peaks occurred displacement from 3392 to $3323 \mathrm{~cm}^{-1}$. The relative strength of absorption peaks of collagen amide I band, II band, and III band were weakened, which indicated that the IL had an effect on the three-dimensional structure of collagen, peptide chains, and peptide bonds.
Above analysis showed that the internal hydrogen bond of undissolved bone became weak after the IL dissolved the bone, and also the relative intensity of characteristic peak did in the collagen amide zone. The higher the temperature was, the more thoroughly the IL dissolved bone collagen. This enhanced influence of the IL on the collagen structure, hydrogen bond, and molecular groups in peptide chain $[22,23]$. So the bone collagen was dissolved mainly in the IL. This was consistent with HSPOM observations. The change of internal hydrogen bond was an important factor of the IL dissolving bone.

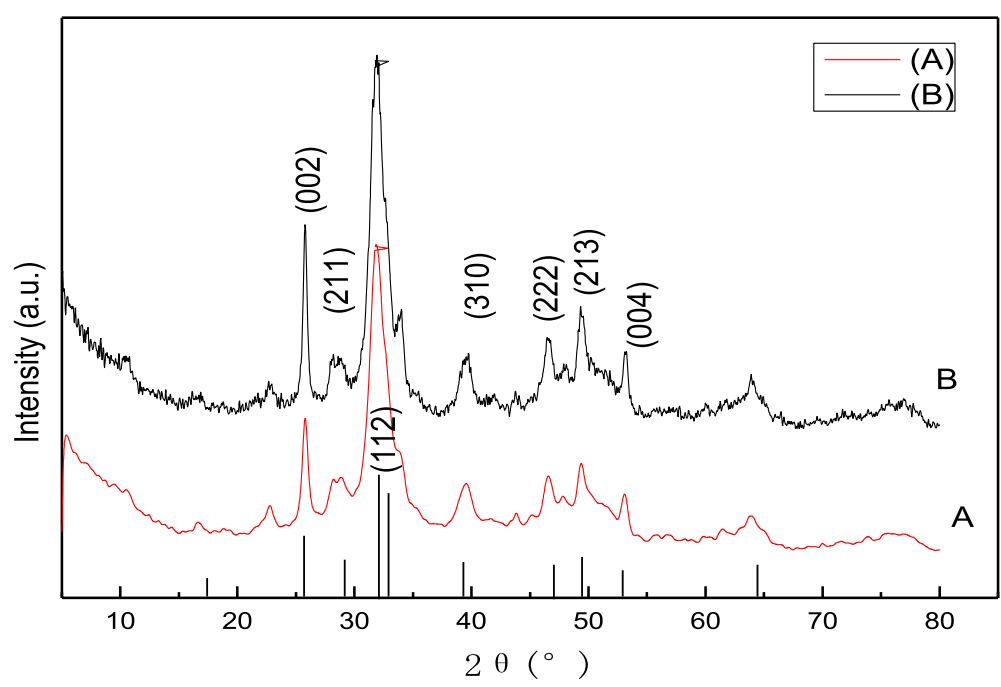

Fig. 5 XRD pattern of sample (A) and extracted (B) HAp 


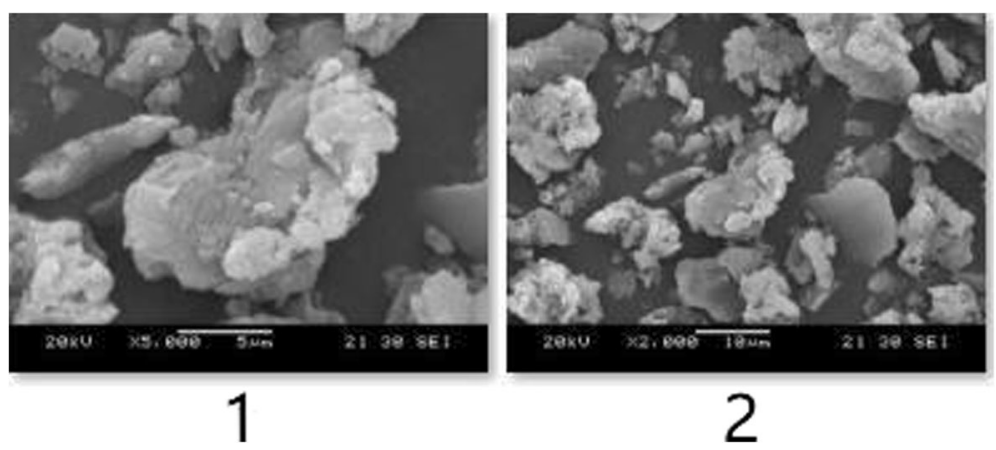

Fig. 6 SEM images of extract HAp (left. magnified 5000 times, right: 2000 times)

\subsection{Characterization and performance analysis of extract HAp}

\subsubsection{FTIR characterization}

Figure 4 was IR spectra of the sample HAp and the extracted HAp. There was a wide absorption peak between 3600 and $3200 \mathrm{~cm}^{-1}$, which was caused by -OH stretching vibration peak of hydrogen-bond association and absorption peak of adsorption water on the surface of extracted HAp. The weak absorption peak at 1619 or $1667 \mathrm{~cm}^{-1}$ explained that the surface of extracted HAp adsorbed water molecules. The absorption peak at 1444 or $1468 \mathrm{~cm}^{-1}$ implied that CO32- entered the HAp structure and replaced some PO43-. The absorption peaks of carbonate or free radical $\mathrm{CO} 32$ - were single peaks at $1400-1500 \mathrm{~cm}^{-1}$. When the CO32- entered into HAp lattice and replaced the PO43-group in the lattice, the absorption peaks appeared splits. The strong absorption band at 1035 or $1031 \mathrm{~cm}^{-1}$ was stretching vibration peak of the group PO43-. The absorption peaks of 604 and $563 \mathrm{~cm}^{-1}$ were related to the bending vibrational absorption peaks of the group PO43-. HAp structure of the sample and extracted HAp were basically the same. The only difference between was absorption peak disappearing at $2921 \mathrm{~cm}^{-1}$. The absorption peak at $2921 \mathrm{~cm}^{-1}$ was also -OH stretching vibration peak of hydrogen-bond association, which indicated that hydrogen bond structure of the product HAp was not damaged in dissolving process. IR characterization indicated that the extracted HAp had the macroscopic composition and microstructure of natural bone [24]. That is, the product HAp has application value, which the process of IL dissolving animal bone can prepare HAp.

\subsubsection{XRD characterization}

Figure 5 shows X-ray diffraction diagrams of sample (A) and extracted (B) HAp. It could be seen that the characteristic diffraction peak of the product HAp was almost completely the same with the standard sample. There was no other impurity peak, which meant that purity of the extracted HAp was high. Although three overlapping peaks were not clearly separated, the peaks were sharp. These explained the crystallinity of the extracted HAp better in this experiment $[25,26]$, having the macroscopic composition and microscopic structure of natural bone. Moreover, its purity is higher than the sample's. That implied that the process of IL directly dissolving biological bones could be used for preparation of HAp.

\subsubsection{SEM characterization}

Figure 6 was scanning electron micrographs of the product HAp, which were magnified 5000 and 2000 times respectively. Two pictures showed that diameters of crystal particles were 5 and $10 \mu \mathrm{m}$, and the different forms of particles could be seen. Most of the particles belonged to micron-sized and even tinier, but their crystal structures were relatively complete. The tiny grain morphology was consistent with the broad peak above XRD pattern.

\subsubsection{Bulk density and BET characterization}

The average value of bulk density of the product HAp was $2.93 \mathrm{~g} / \mathrm{cm}^{3}$, which was shown in Table 7 . This density was slightly smaller than $3.16 \mathrm{~g} / \mathrm{cm}^{3}$ of the sample, which had little difference. According to the measured values of BET in Table 7, specific surface area, pore

Table 7 Bulk density and BET data

\begin{tabular}{|c|c|c|c|c|c|}
\hline & $\begin{array}{l}\text { Bulk density } \\
/ \mathrm{g} / \mathrm{cm}^{3}\end{array}$ & $\begin{array}{l}\text { Specific surface } \\
/ \mathrm{m}^{2} \mathrm{~g}^{-1}\end{array}$ & $\begin{array}{l}\text { Pore volume } \\
/ \mathrm{cm}^{3} \mathrm{~g}^{-1}\end{array}$ & $\begin{array}{l}\text { Pore diameter } \\
\text { /nm }\end{array}$ & $\begin{array}{l}\text { Grain size } \\
/ \mathrm{nm}\end{array}$ \\
\hline Product HAp & 2.93 & 58.72 & $0 . .46$ & 14.27 & 34.87 \\
\hline Sample HAp & 3.16 & 56.77 & 0.19 & 13.60 & 33.0 \\
\hline
\end{tabular}

BET grain size is calculated on the basis of $D=6 /\left(\rho \cdot S_{B E T}\right)$, where $D$ is the grain size of HAp particle $(\mathrm{m})$

$\rho$ the density $(\mathrm{g} / \mathrm{cm} 3)$; SBET the specific surface area $(\mathrm{m} 2 \cdot \mathrm{g}-1)$ 


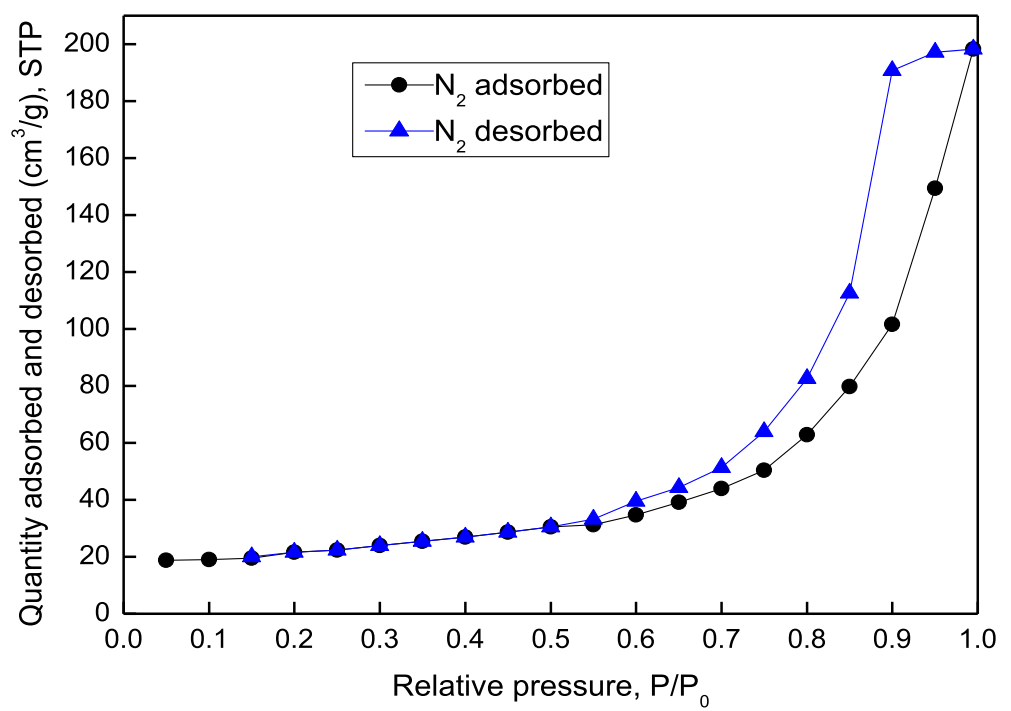

Fig. 7 N2 adsorption-desorption isotherm of extracted HAp

volume, and pore diameter of the product HAp were similar to ones of the sample HAp. Based on classification of pore diameter: micropore $(<2 \mathrm{~nm})$, mesoporous $(2 \sim 50 \mathrm{~nm})$, macropore $(>50 \mathrm{~nm})$, the extracted HAp pertained to mesoporous due to the pore diameter value of $14.27 \mathrm{~nm}$ [27].

Figure 7 displayed N2 adsorption-desorption curves of the extracted HAp. It showed that the product had a significant surface area. The larger the surface area is, the stronger the surface effect, such as surface activity, surface adsorption capacity, catalytic ability, and so on. So, this product could be used for different adsorption process, especially for the drug load in the treatment of bone-related diseases due to playing a good role in drug release carrier [28].

\subsubsection{Thermogravimetric analysis}

Figure 8 shows TG curve of the extracted HAp, which declined slowly when temperature rose from 0 to $200^{\circ}$ C. It explained that the residual moisture of HAp flowed away gently. As the temperature increases from 200 to $450{ }^{\circ} \mathrm{C}$, three helical structures of collagen in HAp were damaged, and TG curve dropped sharply, which made collagen gradually reduced. When the temperature continuously rose from 450 to $1200{ }^{\circ} \mathrm{C}$, the TG curve declined gradually trending to a smooth and steady phase. This indicated that thermal decomposition temperature

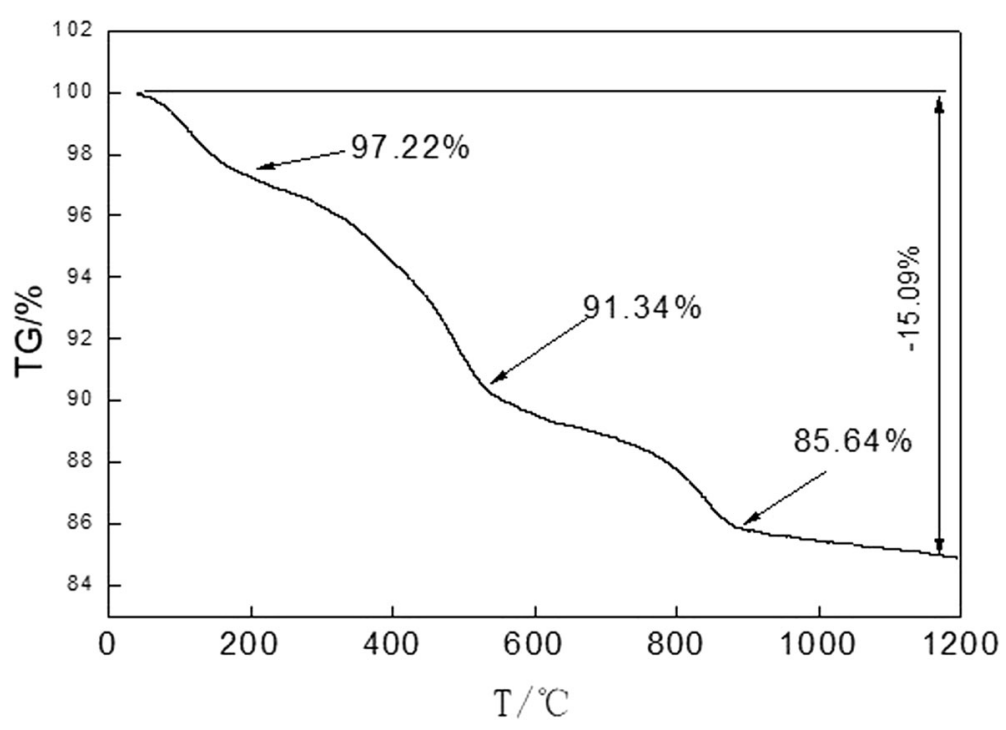

Fig. 8 The TG curve of extracted HAp 


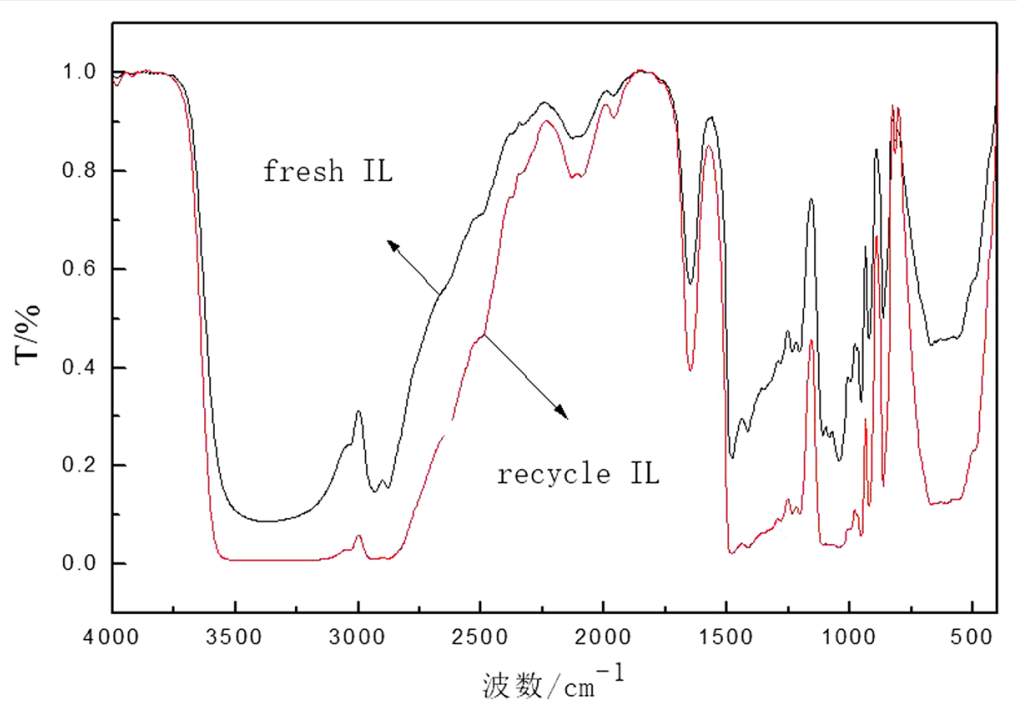

Fig. 9 IR spectra of fresh and recycle IL

of the extracted HAp was higher, so its stability was better. Its overall weight loss rate was $15.09 \%$.

\subsubsection{Recycle IL}

The IL was recycled three times. The recycle rates were 94.7, 93.3, and 95.8\% respectively. The second recycled IL was condensed on the rotary evaporation apparatus under $70{ }^{\circ} \mathrm{C}$, and dried in the drying oven after evaporated water. The recycled IL was characterized by IR and contrasted with synthetic IL (fresh). Their infrared spectra in Fig. 9 showed structures of the IL before and after recycle were basically the same. The result indicated that the ionic liquid still retained its original structure after recycling and could be reused.

\section{Conclusion}

The dissolving process of chicken bone in ionic liquid (IL) choline chloride-glycerol (ChCl-GL) was researched, and the product hydroxyapatite (HAp) was extracted. The optimum conditions of extracted HAp were determined by single factor, orthogonal, and verification tests. Based on dissolving situation of observing bone power in the IL by means of thermal stage polarized optical microscope (HSPOM), it could be preliminarily concluded that this dissolving process was a direct physical dissolution. IR analyses, which was for undissolved bone, under different solution temperature and time, showed that bone collagen was dissolved mainly in the IL. This was consistent with HSPOM observations. The change of internal hydrogen bond was an important factor of IL dissolving bone.

FTIR characterization indicated that the extracted HAp had the macroscopic composition and microstructure of natural bone. XRD and SEM displayed that the product was much similar to the standard sample, and its crystal structure was relatively complete; moreover, its purity is higher than the sample's. Most of particles belonged to micron-sized and even tinier.

The BET tests exposed that specific surface area and aperture of the extracted HAp were similar to the value of the sample, which belonged to the mesoporous. Analysis of N2 adsorption-desorption revealed that the extracted HAp had a significant specific surface area, which could be used for different adsorption processes, especially for the drug load in the treatment of bone-related diseases due to playing a good role in drug release carrier. TG characterization exposited that pyrolysis temperature of the extracted HAp was higher, so it had better stability. $\mathrm{IL}(\mathrm{ChCl}-\mathrm{GL})$ could be reused and its structure still retained its original form after recycling.

\section{Abbreviations}

BET: Brunauer-Emmett-Teller; ChCl-GL: Choline chloride-glycerol; FTIR: Fourier transform infrared spectroscopy; HAp: Hydroxyapatite; HSPOM: Thermal stage polarized optical microscope; IL: Ionic liquid; SEM: Scanning electron microscope; XRD: X-ray diffraction

\section{Acknowledgements}

The authors thank the editor and anonymous reviewers for their helpful comments and valuable suggestions.

\section{Funding}

This work was supported in part by a grant from the National Natural Science Foundation of China (No.21476175).

Availability of data and materials We can provide the data.

\section{Authors' contributions}

All authors take part in the discussion of the work described in this paper. The author $\mathrm{HZ}$ wrote the first version of the paper, The author YD did part experiments of the paper, WS revised the paper in different version of the paper. All authors read and approved the final manuscript. 


\section{Authors' information}

Hong Zhu received the BS degree in inorganic non-metallic materials in 1992 from Nanjing University of Technology, Nanjing China. She is an associate professor of the Institute of Biology and Environment Engineering, Tianjin Vocational Institute. Her research focuses on biochemistry, Material science, and Environmental engineering.

Wei Song received the BS degree in Chemical Engineering in 1983 and MS degree in Industrial Catalysis in 1996 from Tianjin University, Tianjin, China. From 1996 to 2000, she was an assistant professor in Tianjin Chemical Engineering School. From 2001 to now, she is a professor of Tianjin University of Technology. Her research interests include Material Science, Chemical Engineering, and Environmental engineering

Yu Deng received his B. S. from the College of Chemical Engineering, Tianjin University in 1983. He is a Professor at the School of Materials Science and Chemical Engineering, Tianjin University of Science \&Technology. His current research interests include Biological materials, lonic liquids, and Chemical Engineering.

\section{Ethics approval and consent to participate} Approved.

\section{Consent for publication}

Approved.

\section{Competing interests}

The authors declare that they have no competing interests.

\section{Publisher's Note}

Springer Nature remains neutral with regard to jurisdictional claims in published maps and institutional affiliations.

\section{Author details}

${ }^{1}$ Institute of Biology and Environment Engineering, Tianjin Vocational Institute, Tianjin 300410, China. ${ }^{2}$ Tianjin Key Laboratory for Advanced Mechatronic System Design and Intelligent Control, School of Mechanical Engineering, Tianjin University of Technology, No. 391 Binshuixi Road, Tianjin 300384, China. ${ }^{3}$ School of Materials Science and Chemical Engineering, Tianjin University of Science \&Technology, Tianjin 300457, China.

Received: 8 April 2018 Accepted: 25 June 2018

Published online: 10 July 2018

\section{References}

1. YJ Guo, WH Wen, Research process of biological engineering on bone defect repairing. Chin. J. Osteoporos. 20(08), 988-993 (2014)

2. C Du, NR Zhao, JD Ye, XF Chen, YJ Wang, Biomimetic functionalized bone repair materials. Mater. China 31(5), 2-10 (2012)

3. JG Liao, YQ Li, XZ Duan, LG Zhu, Nana-hydroxyapatite/polymer composite as bone repair materials. Prog. Chem. 27(2/3), 220-228 (2015)

4. $\mathrm{K} \mathrm{Hu}, \mathrm{Y}$ Zhang, WW Ren, Current approaches of artifical bone repair materials - hydroxyapatide/polylactide composite. Chin. Orthop. J. Clin. Basic Res. 5(1), 56-62 (2013)

5. Y Jin, HX Guo, The preparation and development prospects of hydroxyapatite biomaterials. Guangdong Chem. Ind. 12(42), 88-89 (2015)

6. AL Gavrilovic, A Piattelli, K Abe, Nerve growth factor beta(NGF beta) delivery via a collagen/hydroxyapatite (Col/HAP) composite and its effects on new bone growth. J. Mater. Sci. Mater. Med. 14(2), 95-102 (2003)

7. L Chen, Y Chen, ZL Wang, Preparation of collagen/hydroxyapatite composites and its research in the repair for bone defect. Chin. Inj. Repair Wound Healing (Electron. Ed.) 11(3), 232-235 (2016)

8. A Joseph Nathanael, SI Hong, D Mangalaraj, PC Chen, Large scale synthesis of hydroxyapatite nanospheres by high gravity method. Chem. Eng. J. 173, 846-854 (2011)

9. Y Daniels, N Lyczko, A Nzihou, D Spiro, Alexandrators. Modification of hydroxyapatite with ion-selective complexants: 1-Hydroxyethane-1,1diphosphonic acid. Ind. Eng.Chem. Res. 54, 585-596 (2015)

10. M Karimi, S Hesaraki, M Alizadeh, A Kazemzadeh, One-pot and sustainable synthesis of nanocrystalline hydroxyapatite powders using deep eutectic solvents. Mater. Lett. 175, 89-92 (2016)

11. A Stark, lonic liquid in the biorefinery: a critical assessment of their potential. Energy Environ. Sci. 4, 19-32 (2011)
12. G Totaro, P Marchese, L Sisti, A Celli, Use of ionic liquids based on phosphonium salts for preparing biocomposites by in situ polymerization. J. Appl. Polym. Sci. (2015). https://doi.org/10.1002/APP.42467

13. M Taha, MR Almeida, FA Silva, Novel biocompatible and self-buffering ionic liquids for biopharmaceutical applications. Chem. Eur. J. 21, 47814788 (2015)

14. FF Fu, Y Deng, BZ Cheng, Dissolution characteristics and comparison of collagen fiber in imidazole ionic liquids. China Leather 39(23), 21-24 (2010) 39(7), 22-25 (2010)

15. H Zhang, J Wu, J Zhang, et al., 1-Allyl-3-methylimidazolium chloride room temperature ionic liquid:A new and powerfulnon-derivatizing solvent for cellulose. Macromolecules 38(20), 8272-8277 (2005)

16. DM Phillips, LF Drummy, DG Conrady, Dissolution and re-generation of bombyxmori silk fibroin using ionic liquids. J. Am. Chem. Soc. 126(44), 14350-14357 (2004)

17. HP Ren, SQ Sun, L Li, Dissolution performance of ionic liquid for silk fibroin. Appl. Chem. Ind. 43(7), 1255-1257 (2014)

18. C Zheng, Study of dissolution and rheology of collagen/ionic liquids solutions (South China University of Technology, Guang Zhou, China, 2013)

19. XC Yuan, PF Wang, FB Yang, Y Deng, Study on dissolving technology of grinding bone in ionic liquid. Hangzhou Chem. Ind. 47(1), 18-22 (2017)

20. N Muhammad, YA Gao, Extraction of biocompatible hydroxyapatite from fish scales using novel approach of ionic liquid pretreatment. Sep. Purif. Technol. 161, 129-135 (2016)

21. $Y Y$ Zhang, $X Y$ Ji, $X H$ Lu, Properties and applications of choline chloride/urea and choline chloride/glycerol. Sci. China Chem. 44(6), 927-941 (2014)

22. YW Zhou, BZ Cheng, Y Deng, HP Shang, Dissolution characteristics of collagen fiber in ionic liquid. China Leather 39(7), 22-25 (2010)

23. ZM Wang, W Liao, L Li, C Zheng, Dissolution and rheological behavior of collagen in ionic liquids. Mod. Food Sci. Technol. 30(11), 34-37 (2014)

24. YM LV, LM Cheng, GX Pei, Characterization and biocompatible evaluation of nHA/RHLC/PLA composite scaffold. Chin. J. Clin. Anat. 29(5), 550-556 (2011)

25. CA Maxwell, TJ Wess, CJ Kenned, X-ray diffraction study into the effects of liming on the structure of collagen. Biomacromolecules 7(8), 23212326 (2006)

26. N Wu, KY Wang, Effect of $\mathrm{pH}$ on preparation and characterization of nanohydroxyapatite-gelation composite. Leather Sci. Eng. 23(4), 5-8 (2013)

27. ZM Guo, XM Chen, XN Li, Preparation and characterization of hydroxyapatite hollow nanoparticles. Guangzgou Chem. 38(5), 142-143 (2010)

28. RR Bi, HT Ding, LJ Wan, Hydroxyapatite and its Application in Biomedicine: preparation and structure. Chem. Bull. (4), 291-296 (2010)

\section{Submit your manuscript to a SpringerOpen ${ }^{\circ}$ journal and benefit from:}

- Convenient online submission

Rigorous peer review

- Open access: articles freely available online

- High visibility within the field

Retaining the copyright to your article

Submit your next manuscript at $>$ springeropen.com 